\title{
Critical evaluation of American categorization of fetal heart rate (FHR) decelerations and three tier classification -Shortcomings, contradictions, remedies and need for debate*
}

\author{
Shashikant L. Sholapurkar \\ Department of Obstetrics and Gynaecology, Royal United Hospital Bath NHS Trust, Bath, UK \\ Email: s.sholapurkar@nhs.net
}

Received 20 February 2013; revised 22 March 2013; accepted 30 March 2013

Copyright (C) 2013 Shashikant L. Sholapurkar. This is an open access article distributed under the Creative Commons Attribution License, which permits unrestricted use, distribution, and reproduction in any medium, provided the original work is properly cited.

\begin{abstract}
Fetal heart rate (FHR) decelerations are the commonest aberrant feature on cardiotocograph (CTG) thus having a major influence on classification of FHR patterns into the three tier system. The unexplained paradox of early decelerations (head compressionan invariable phenomenon in labor) being extremely rare [1] should prompt a debate about scientific validity of current categorization. This paper demonstrates that there appear to be major fallacies in the pathophysiological hypothesis (cord compressionbaroreceptor mechanism) underpinning of vast majority of (variable?) decelerations. Rapid decelerations during contractions with nadir matching peak of contractions are consistent with "pure" vagal reflex (head compression) rather than result of fetal blood pressure or oxygenation changes from cord compression. Hence, many American authors have reported that the abrupt FHR decelerations attributed to cord compression are actually due to head compression [2-6]. The paper debates if there are major fundamental fallacies in current categorization of FHR decelerations based concomitantly on rate of descent (reflecting putative aetiology?) and time relationship to contractions. Decelerations with consistently early timing (constituting majority) seem to get classed as "variable" because of rapid descent. A distorted unscientific categorization of FHR decelerations could lead to clinically unhelpful three tier classification system. Hence, the current unphysiological classification needs a fresh debate with consideration of alternative models and re-evaluation of clinical
\end{abstract}

*Declaration of interests: The author has no conflict of interest to declare. No funding was received. studies to test these. Open debate improves patient care and safety. The clue to benign reflex versus hypoxic nature of decelerations seems to be in the timing rather than the rate of descent. Although the likelihood of fetal hypxemia is related to depth and duration of FHR decelerations, the cut-offs are likely to be different for early/late/variable decelerations and it seems to be of paramount importance to get this discrimination right for useful visual or computerized system of CTG interpretation.

Keywords: Cardiotocography; Electronic Fetal Monitoring; Fetal Heart Rate Decelerations; Intrapartum Fetal Monitoring; Intrapartum Fetal Surveillance

\section{INTRODUCTION}

American Congress of Obstetricians and Gynecologists (ACOG) commented that a standardized approach to nomenclature, systems of interpretation and management algorithms of fetal heart rate (FHR) abnormalities has remained problematic [7]. Recently, many other countries have based their CTG interpretation systems on American practice. Three tier system of interpretation of FHR tracings have been adopted by all national guidelines but its clinical efficacy has been found significantly wanting in the USA [1,8]. Hence a 5-tier system of classification has been proposed $[1,8]$ which is quite complex, cumbersome and somewhat impractical. It is a clinical observation that FHR decelerations are the most common deviant feature on the cardiotocograph (CTG) quite often in the absence of other FHR abnormalities and hence their interpretation has the greatest influence on the categorization of CTG into the three tier system [7]. Thus categorization of FHR decelerations will often 
make or break CTG interpretation. This clinical opinion paper has a limited scope with focus on decelerations only and other less controversial FHR changes like baseline rate and accelerations are not the subject. In USA, a major drawback is that almost $80 \%$ of all FHR tracings fall in the Category II of indeterminate significance in the three tier system [8]. "Early" decelerations are extremely rare [1] and all other FHR decelerations (late and all variable decelerations) fall in the category II. The clinician can "continue to observe”, "evaluate further” or "deliver" on individualized basis as no management algorithm can be prescribed for Category 2, which has been a major criticism [8]. However, this apparent lack of discriminatory potential may not be due to the three tier system itself but could be because of how different types of FHR decelerations are interpreted or categorized. It is important to note that all classifications of FHR decelerations are mainly based on level IV evidence. Although level 1 or 2 evidence is lacking for most aspects of electronic fetal monitoring (EFM), this need not be a complete impediment for this clinical opinion paper which is based on step wise logical constructs, physiological principles and common clinical observations. The main aim of this clinical opinion paper is to encourage a debate about theoretical hypotheses underpinning evolution of American classification of FHR decelerations, any contradictions, clinical implications and alternative systems of classification. Open debate facilitates scientific progress and enhances patient care and safety.

\section{PIONEERING WORK BY EDWARD HON IN USA}

Hon and Quilligan (1968) in their final paper described three FHR deceleration patterns primarily based on whether they had their onset at the beginning (early decelerations), 20 - 30 seconds after beginning (late decalerations) of contractions or if onset time was variable (variable decelerations) [9]. Purely as preliminary hypotheses, Edward Hon somewhat ambiguously described "early" decelerations to have a "uniform FHR pattern" of a specific shape whose onset almost coincides with beginning of contraction and whose waveform reflects (somewhat vague term in itself) the associated amniotic fluid pressure curve. However, he added that this reflection may not be apparent anyway because of scale used for display. The speculation that "early" and "ate" decalerations should somehow "reflect” the "apparent" gradual shape of uterine contraction curve (now more commonly recorded by external pressure transducer in many countries) seems to have taken root in North America without any scientific studies. Even if contraction curves "look" gradual, it does not necessarily follow that the consequent rise in fetal intracranial pressure and resultant FHR drop due to direct reflex parasympathetic (vagal) suppression of SA node should also be gradual. "Early" decelerations have remained very rare in American practice precisely because of the requirement for them to be "gradual" in shape despite several contrary observations of rapid head compression decelerations by many American authors including by Hon himself [2-6]. Both head compression as well as cord compression could cause rapid decelerations. Moreover, work by Cibils [10] and Krebs [11] in USA seem to have consolidated a hypothesis/belief that rapid/abrupt FHR decelerations are synonymous with "umbilical dips" or "cord compression decelerations" and that an initial acceleration foretells cord compression. Prevailing thinking has been that when only umbilical venous compression occurs, reduction in venous return causes initial tachycardia followed by eventual abrupt deceleration due to subsequent hypoxemia; whereas predominant umbilical arterial compression causes rapid deceleration due to resultant hypertension. However, no attention seems to have been paid to the onset of recovery of these decelerations. Both venous and arterial compression are maximal or submaximal close to the height of contraction and thus any FHR decelerations that start recovering close to height of contraction cannot be explained by "cord compression" as discussed in details later.

\section{CURRENT NORTH AMERICAN DEFINITIONS OF FHR DECELERATIONS}

ACOG adopted guidelines including definitions of FHR decelerations proposed by the National Institute of Child Health and Human Development (NICHD) [7,12]. NICHD stated that they attempted to rigorously and unambiguously standardize definitions of FHR patterns and no prior assumptions were made about their relationship to fetal hypoxemia or acidemia [12]. The other assertion by NICHD that no prior assumptions were made about putative aetiology is debatable because decelerations were classified based on gradual or rapid descent which seemingly draws from longstanding unproven beliefs about effects of head/cord compression, unless there is some other explanation.

It was observed by Hon and Quilligan (1968) that FHR decelerations lend themselves very well to be classified according to their time relationship to contractions and confirmed recently as the most commonly used system in USA $[9,13]$. However, whether decelerations lend themselves to be divided into meaningful groups based on rate of descent is not clear and needs to be debated. It can indeed be demonstrated that combining the two systems leads to significant inconsistencies [14]. The most obvious contradiction is that decelerations with consistently early timing (constituting majority) get classed as "variable" based on rapid descent. Practical interpreta- 
tion of ACOG definitions [15] shows that the first and foremost criterion is "time of descent" which takes precedence over all other parameters including time relationship to contractions. Thus the three categories of FHR decelerations by ACOG could more aptly be described as "rapid", "gradual early" and "gradual late". The old terminology of "early/late/variable" decelerations does not seem to accurately describe this classification anymore.

To avoid subjective inter-observer variation in differentiating "gradual" decelerations from "rapid" ones, NICHD seems to have come up with an arbitrary "30 seconds cut-off" for time taken by FHR to reach nadir from the baseline [12]. This is a proxy for "slope" of the deceleration waveform which cannot be calculated and used easily in practice. "Variable" decelerations were defined as those with time of descent to be less than 30 seconds (rapid). "Early" and "late" decelerations were defined to have descent time of more than 30 seconds (gradual) and "usually symmetrical" in shape [7,12]. Thus rapid decelerations even with onset consistently "early" in the contractions and recovering before the end of contractions will still be classed as "variable". However, there is no evidence that this cut-off of 30 seconds or indeed the rate (slope)/time of descent are helpful in discriminating healthy from hypoxemic fetuses. Parer and Ikeda reported that fetal academia was best related to duration and depth of decelerations irrespective of rapid or gradual descent [1]. More importantly this arbitrary "30-second cut-off" leads to very strange aberrations described below [14].

\section{EARLY DECELERATIONS AND HEAD COMPRESSION}

Fetal head compression is almost universal phenomenon in labor and hence it is surprising that there are hardly any early decelerations when NICHD/ACOG definitions are followed [1]. The reasons may lie in a flawed speculation that "early" decelerations must have gradual descent. It is a practically verifiable observation that the recovery time of FHR deceleration (early and late decalerations symmetrical as per ACOG) is at least same or often a bit longer than the time for descent. Hence, any decelerations with descent time of more than 30 seconds would necessarily be of more than 60 - 65 seconds duration. Thus "early" decelerations based on ACOG definitions will always have to be of more than 60 seconds duration which is paradoxical for supposedly completely benign decelerations. There is no rational reason why any class of decelerations must always be more than 60 65 seconds in duration. This aberration is not just a theoretical issue but is likely to distort the interpretation of decelerations with resultant loss of discriminatory potential. Many recorded contraction curves last for less than 60 seconds on CTG. All decelerations occurring during these contractions will always be classed as "variable" by default. These contractions simply cannot have "early" decelerations by the very accident of definition which does not seem logical or scientific. Thus "early" decalerations (the most benign class) would become almost impossible to find as a direct paradoxical consequence of ACOG definitions. Many American Obstetricians classify decelerations of 40 - 60 seconds duration with consistently late timing as "late" decelerations (personal correspondence) probably quite rightly; but this would be contradictory to ACOG definitions as the "time of descent" would be less than 30 seconds. It needs to be debated if these contradictions are in conflict with basic scientific principles or are fundamental enough to warrant going back to the drawing board.

There have been controversies about definition of early decelerations in the past $[1,14]$. Characterized by gradual descent they have been proposed to occur between 4 and $7 \mathrm{~cm}$ dilatation $[3,16,17]$ although head compression would be more common and pronounced in late first and second stage of labor. Ball and Parer concluded that decelerations due to head compression have rapid descent and are more common in second stage [2]. They remarked that head compression is the likely cause of many variable decelerations classically attributed to cord compression, an observation also made by Garite [2,3]. Studies by group of Caldeyro-Barcia, Rech, and Chung and Hon demonstrated that manual trans-abdominal and vaginal compression of fetal head resulted in rapid FHR decelerations [4-6]. Hence it can be concluded that it seems unscientific to define early decelerations as "gradual" based on putative aetiology or arbitrarily or for any other reason especially when a direct consequence is to make a class of most benign decelerations very hard to find. Rate or time of descent does not seem to differentiate between head or cord compression.

Alternative models of classification of FHR decelerations have been practised. For example, in the United Kingdom, expert opinion in mainstream British Textbooks reflected as well as guided the classification of FHR decelerations from 1970s until very recently [1821]. These authorities classified all decelerations starting at the beginning of contraction and recovering before the end of contraction as "early" irrespective of "rate of descent” (Figure 1). This made "early decelerations" to be the majority in British Obstetric practice over many decades $[14,22,23]$. This conforms to physiological expectation that vast majority of FHR decelerations should be benign and a small number are likely to be associated with fetal hypoxemia. Thus the British classification which classed majority of decelerations in to a benign class can be expected to be advantageous in interpreting CTG for discerning fetal status. The Royal College of Obstetricians and Gynaecologists (RCOG) of UK published 


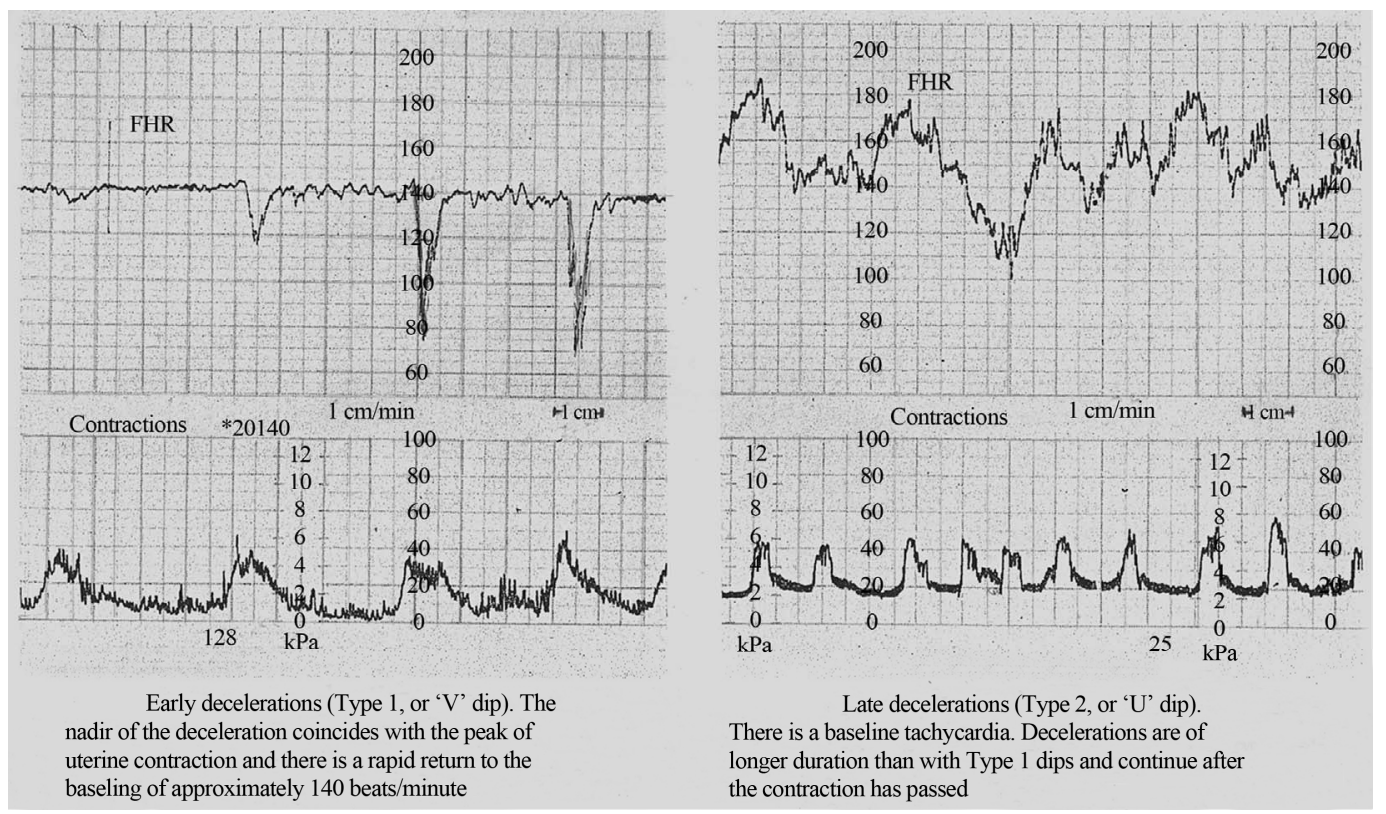

Figure 1. Early and late decelerations in traditional British practice (Reproduced from Textbook of Obstetrics by Bryan Hibbard, 1988) [18]. Note rapid (less than 30 seconds) rather than gradual descent of early as well as some of the late FHR decelerations (CTG paper speed $1 \mathrm{~cm} / \mathrm{min}$ ).

their first guidelines on EFM in 2001 confirming that early decelerations (as interpreted in British practice) were not associated with fetal hypoxemia with a high reliability based on previous studies and expert consensus [24].

\section{Late Decelerations}

There is generally high agreement in all national guidelines and published studies that late decelerations are associated with a significant likelihood of fetal hypoxemia and acidemia [7,17,24,25]. As a direct result of NICHD and ACOG definitions (similar to early decalerations) late decelerations cannot be of less than 60 - 65 seconds duration for no logical or scientific reason whatsoever. Hence many FHR decelerations of less than 60 - 65 seconds with consistently late timing (with possibility of hypoxemia) may get inappropriately categorized into indeterminate class of "variable" decelerations. Thus it may be more scientific to define late decelerations based only on their time relationship to contractions. Further research is required to improve positive predictability of late decelerations to diagnose fetal hypoxemia.

\section{VARIABLE DECELERATIONS AND CORD COMPRESSION}

This is reported to be the most common deceleration pattern in labor in USA and is attributed to umbilical cord occlusion and used to be called "cord compression pattern" $[10,11,13]$. Since umbilical cord lacks parasympathetic and sympathetic innervation [26,27], cord com- pression by itself (unlike head compression) cannot elicit a "direct" or "pure" vagal reflex producing FHR drop which would simulate inverted image of a contraction. Hence, different permutations and combinations of baroreceptor and chemoreceptor mechanisms and partial/ complete cord compressions have been proposed as underlying pathophysiological mechanism [13]. Lee et al. (1975) and many other authors proposed initial venous occlusion leading to loss of fetal blood volume (through patent umbilical artery) triggering initial FHR acceleration or shoulder [13,17,28-30]. To explain subsequent rapid FHR drop via baroreceptor mechanism, complete arterial occlusion (complete cord compression) will be required and has indeed been proposed causing fetal systemic hypertension [13,17,18,28-30] (Figure 2). How likely is it that the cause of vast majority of FHR decelerations in labor is "complete" cord compression rather than head compression? Transient hypoxemia is another mechanism proposed $[11,13]$. Indeed many animal studies have shown that hypoxia through chemoreceptor mechanism seems to be more dominant cause of FHR decelerations associated with cord compression [31,32] but these are likely to be late in timing or at least late to recover (i.e. after relief of venous compression). Lastly, baroreceptor mechanism proposes recovery of FHR following relief of umbilical artery occlusion but illustrations $[13,17,28-30]$ show this occurring close to or at the peak of contractions (Figure 2) where FHR recovery starts. It is clearly not possible that umbilical arterial occlusion which is proposed to occur in the early part of contraction would be consistently relieved at the peak of 


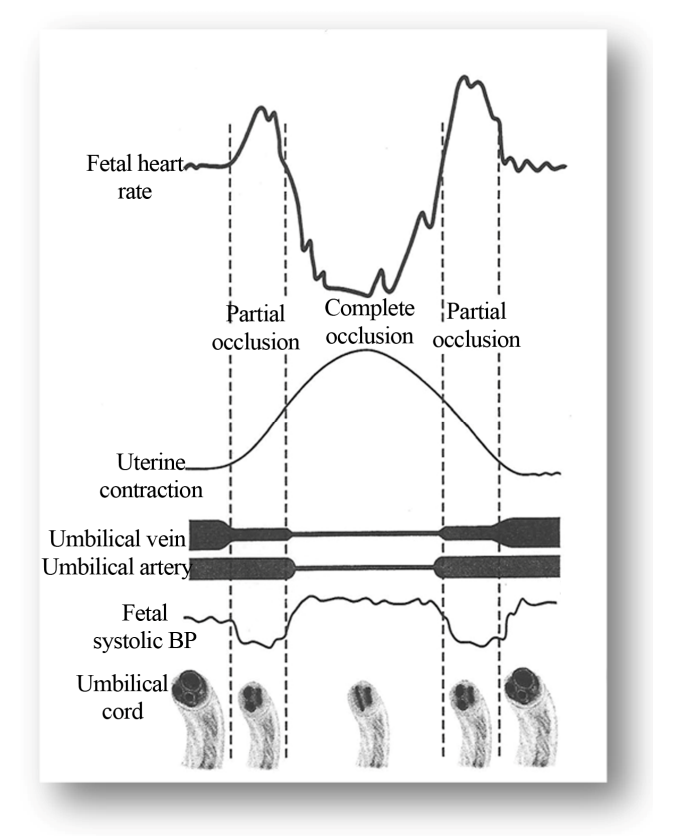

Figure 2. Schematic representation of the hypothesis of "FHR variable deceleration and cord compression", adapted from Williams Obstetrics (2010) and Lee et al. (1975) [13,28]. This hypothesis is the mainstay of categorization of vast majority of FHR decelerations as "variable" (result of cord compression). However, this hypothesis seems to have major flaws. Note the FHR recovery starting at the height of contraction which cannot be explained by the hypothesis illustrated and is contradictory to theoretical modeling (see text) and evidence from animal studies [31-35]. The deceleration depicted seems consistent with "direct" or "pure" vagal reflex (head compression rather than cord occlusion).

contractions. Umbilical arterial compression could cause arterial hypertension but this does not reverse at the peak of contraction. Even more importantly, many animal experiments show that FHR bradycardia precipitated by short term (about a minute or so) complete or partial cord occlusion starts recovering only after relief of complete and partial cord occlusion [31-35] which would be much later after peak of contraction. Thus theoretical modeling as well as substantial evidence from many animal experiments proves that cord compression cannot explain the most common "apparent" rapid FHR decelerations during contractions with the trough of deceleration consistently corresponding to the peak of contraction (Figure 2). These decelerations are consistent with "direct" or "pure" vagal reflex, the commonest cause being head compression (and possibly multifactorial) as indeed proposed by many authors [2,4-6,18-21] and hence would be best classed as early decelerations. This would be consistent with description by Hon [9], Krebs [11] and most British authors [18-21] that variable decelerations would be primarily characterized by variation in timing. The small accelerations (shoulders) need not always be a result of cord compression but may simply be a reflection of balance between sympathetic and parasympathetic systems signifying fetal well-being. Lastly, it has been commonly overlooked that simple physical stimulation of the umbilical cord causes direct contraction of musculature of umbilical arteries (not necessarily theoretical venous followed by arterial occlusion) leading to partial constriction [26] but the resultant FHR decelerations would be classically variable in timing and could not be expected to consistently start recovering at the peak of contractions as majority do. Animal experiments suggest that a variety of mechanisms trigger the FHR decelerations with cord compression, including baro-, chemoand mechano-reflex (personal correspondence with Dino A. Giussani, Professor of Developmental Cardiovascular Physiology \& Medicine, University of Cambridge, UK) [31]; and hence are likely to be variable in timing.

Thus based on Hon's description and reasons outlined above, variable decelerations (when defined by variable timing/recovery in relation to contractions) are likely to constitute much small proportion of all decelerations. Further elucidation of variable decelerations remains debatable. "Loss of initial acceleration" is the commonest atypical feature but was found to be relatively benign [11]. On the other hand other atypical features referred by Cibils [10] as "late or hypoxic components" may have better positive predictability. However, when much smaller number of (variable) decelerations will need to be further elucidated in to typical and atypical (abnormal) ones, the overall discriminatory power of different types of FHR decelerations is likely to improve. This is clearly an area for future research. A recent important well designed large study by Cahill et al. (2012) found no correlation between "atypical decelerations" and incidence of neonatal acidemia [36]. They analyzed last 30 minutes of CTG preceding 5388 deliveries and came to a conclusion that the "total deceleration area" was a better predictor of neonatal acidemia [37]. However, one specific feature of this impressive study may be that most CTGs studied were in the second stage of labor. In practice, majority of cases of fetal hypoxia/academia generally commence and need to be detected during the first stage of labor. It is a common clinical observation that the FHR decelerations in second stage of labor (during maternal expulsive efforts) are much deeper and wider compared to the first stage where similar ones would indicate far worse prognosis. Hence in addition to many other unresolved questions, different ranges of "total deceleration area" may have to be defined for first and second stage. In practice, because of the increased frequency and diversity of FHR decelerations in second stage, most senior British Obstetricians consider mainly persistent late decelerations as 
significant. Debate should include whether universal application of same "total deceleration area" parameters to all decelerations (a very heterogeneous group) would be rewarding especially when it represents a complete departure from half a century of relatively well hypothesized and observed importance of "time relationship of decelerations to contraction cycle" [38]. Although the likelihood of fetal hypxemia is related to depth and duration of FHR decelerations [1], the cut-offs are likely to be different for early/late/variable decelerations and it seems to be of paramount importance to get this discrimination right for useful visual or computerized system of CTG interpretation. Unfortunately, American Obstetricians (unlike the British) are unlikely to have had exposure to or a favorable practical experience of a system where "early" decelerations constituted the majority [14]. However, it does appear that a system where vast majority of FHR deceleration are classed as "variable" leads to loss of "information" or discriminatory capacity of FHR decelerations [14,36,37].

\section{Nuchal Cord and Variable Decelerations}

Hankins et al. reported that newborns with a nuchal cord had more variable FHR decelerations in the first and second stage of labor [39]. However, this is not a proof that the prior categorization of decelerations as "variable" (significant cord compression) must be correct. This is because the vast majority (similar proportion) of decelerations were "variable" anyway in the groups with presence and absence of nuchal cord. More importantly, the prevalence of umbilical cord acidemia increased from $11.8 \%$ in controls (somewhat high) to $20 \%$ in the presence of nuchal cord [39]. Hence it seems crucial that an attempt should be made to differentiate benign reflex from hypoxic decelerations rather than placing all decelerations in a single indeterminate category. FHR decelerations with nuchal cord are likely to be multifactorial.

\section{THREE TIER CLASSIFICATION OF FHR PATTERNS}

Three tier systems take in to account many aspects of FHR patterns and hence are likely to be better in predicting fetal status [24]. FHR decelerations do have a maximum and disproportionate influence on them. It follows that inappropriate and unscientific categorization of FHR decelerations is not only of theoretical interest but will impact on ability of three tier systems to predict fetal condition and thus their clinical usefulness. The classification of FHR decelerations based primarily on time relationship to contractions as described by Hon and others [2,4-6,18-21] is likely to be more predictive of fetal condition. This is because they categorize majority into a benign group of "early" (as expected physiologi- cally) and smaller number of decelerations into "late" and "variable" groups. This is exemplified by the British three tier system (RCOG, 2001) in which "early" decelerations are normal, "typical variable” decelerations fall into suspicious category, while "atypical variable” and "late” decelerations (even without FHR baseline abnormalities) fall into pathological category [24]. American three tier system on the other hand has been criticized for lack of usefulness in practice because almost $80 \%$ of all abnormal CTGs fall in to indeterminate category II where no definite management recommendations can be made [8]. This may be a result of vast majority of FHR decelerations being inappropriately classed as "variable" adversely affecting ability of different types of decelerations to predict fetal hypoxemia or well being. A large proportion of fetuses with acidemia will have decelerations but not necessarily absent baseline variability. Thus the category II constitutes large number of benign decelerations as well significant proportion of deceleration patterns associated with acidemia with no practical means to differentiate. Miller and Miller described category II (majority of cases) as “confusing” and proposed a standardized “ABCD” approach to CTG interpretation in general but also aimed to minimize the controversy generated by category II [15]. A "simplified ABCD checklist” constitutes about 25 boxes with several considerations in many individual boxes [15] raising a question whether checklists are always helpful although in fashion. Moreover, there remains lack of clarity and uncertainty about how to determine "clinically significant decelerations” in the large heterogeneous category II group. The solution in "ABCD” approach for this "uncertainty" is to proceed to the next step which is to estimate how long it may take for metabolic academia $(\mathrm{pH}$ below 7.0 in “ABCD” approach) and potential fetal brain injury to develop; and accomplish delivery before this. However, there is no practical guidance possible to decide the potential timing of this eventuality in category II (with either "ABCD” approach or the three tier system); because even the likelihood of this eventuality is the very "uncertainty" in the first place. Moreover, in practice the aim should be to diagnose deceleration patterns associated with $\mathrm{pH}$ of 7.10 or 7.15 and intervene at that stage. Thus the ABCD approach does not seem to solve the problem of confusing and heterogeneous category II. On the other hand, with more logical and judicious categorization of decelerations, late decelerations and variable decelerations with recurrent late components could achieve sufficient discriminatory potential to be classed into Category III similar to British three tier system [24]. Computerized interpretation of FHR patterns is long time coming and proving difficult to be reliable or widely accepted. This again may be because of flawed emphasis on shape of waveform (rapid or gradual) which tends to make cate- 
gorization of decelerations somewhat dysfunctional.

\section{CONCLUSION}

NICHD and ACOG definitions of FHR decelerations based simultaneously on timing as well as shape of waveform (rate/time of descent) lead to conflicting results. Majority of decelerations have consistently early timing but these get classed as "variable" because of rapid descent and putative aetiology. FHR decelerations due to both cord compression and head compression can have rapid descent. Detailed analysis of baroreceptorchemoreceptor hypotheses [14] and evidence from many animal studies [31-35] suggest that rapid decelerations during contractions with trough consistently corresponding to peak of contractions cannot be explained by cord compression. These are consistent with benign direct/ pure vagal reflex mechanism (most likely head compression with some other factors like placental compression). Decelerations due to partial or complete cord compression/mechanical stimulation are almost always likely to have variable/delayed recovery or timing. Moreover, as an unintended but direct consequence, ACOG definitions mandate "early" and "late" decelerations to be always more than 60 - 65 seconds, making early decelerations very rare which is unphysiological and unexplained [14]. It needs to be debated whether the current categorization of FHR decelerations contains any flawed hypotheses, significant contradictions or anomalies undermining basic tenets of scientific practice. Aetiology of FHR decalerations will always remain putative and hence the main aim should be to differentiate between benign reflex versus hypoxic decelerations. FHR decelerations can be said to be of two main types [38], one due to parasympathetic (vagal) reflex and other due to direct suppression of myocardium due to hypoxia. The clue to differentiating this is in the "timing" rather than "rapid or gradual descent”. Since hypoxia during contraction has a lag time to develop or worsen and recover, it causes decelerations which are late in timing by chemoreceptor reflex and in more severe cases by direct myocardial suppression. Fresh debate and re-examination of classification of FHR decelerations is urgently needed. Dysfunctional categorization of FHR decelerations will impair clinical usefulness of CTG interpretation and three tier systems. A recent important research paper by Cahill et al. (2012) also stressed the major influence of FHR decelerations on the 3 tier classification and suggested that the current NICHD nomenclature of decelerations may result in loss of information [37]. Alternative models of classifications should be considered and subjected to expert consensus and clinical studies. Even materials from existing clinical studies could be subjected to reanalysis using different classification of decelerations. It needs to be explored if definitions of FHR decelerations based primarily and solely on time relationship to contractions as originally proposed by Hon and others [2,4-6,18-21] are more scientific and clinically useful.

\section{ACKNOWLEDGEMENTS}

The author greatly appreciates contribution by all clinicians, authors and researchers to the field of EFM to date. The author is thankful to the very helpful and efficient library staff who helped with the literature search.

\section{REFERENCES}

[1] Parer, J.T. and Ikeda, T. (2007) A framework for standardized management of intrapartum fetal heart rate patterns. American Journal of Obstetrics and Gynecology, 197, 26.e1-26.e6.

[2] Ball, R.H. and Parer, J.T. (1992) The physiologic mechanisms of variable decelerations. American Journal of $\mathrm{Ob}$ stetrics and Gynecology, 166, 1683-1688.

[3] Garite, T.J. (2002) Intrapartum fetal evaluation. In: Gabbe, S.G., Niebyl, J.R. and Simpson, J.L., Eds., ObstetricsNormal and Problem Pregnancies, 4th Edition, Churchill Livingstone, New York, 395-429.

[4] Mendez-Bauer, C., Poseiro, J.J., Arellano-Hernandez, G., Zambrana, M.A. and Caldeyro-Barcia, R. (1963) Effects of atropine on the heart rate of the human fetus during labor. American Journal of Obstetrics and Gynecology, 85, 1033-1053.

[5] Rech, W. (1933) Untersuchungen über die Herztätigkeit des Fetus. III. Die Wirkung des Kopfdruckes auf die Frequenz des fetalen Herzchlages. Archives of Gynecology and Obstetrics, 154, 47-57.

[6] Chung, F. and Hon, E.H. (1959) The electronic evaluation of fetal heart rate. I. With pressure on the fetal skull. Obstetrics and Gynecology, 13, 633-640.

[7] American College of Obstetricians and Gynecologists (2009) Intrapartum fetal heart rate monitoring: Nomenclature, interpretation and general management principles. Obstetrics and Gynecology, 114, 192-202.

[8] Coletta, J., Murphy, E., Rubeo, Z. and Gyamfi-Bannerman, C. (2012) The 5-tier system of assessing fetal heart rate tracings is superior to the 3-tier system in identifying fetal acidemia. American Journal of Obstetrics and Gynecology, 206, 226.e1-226.e5.

[9] Hon, E.H. and Quilligan, E.J. (1968) Electronic evaluation of fetal heart rate. IX. Further observations on "pathologic" fetal bradycardia. Clinical Obstetrics \& Gynecology, 11, 145-167. doi:10.1097/00003081-196803000-00009

[10] Cibils, L.A. (1978) Clinical significance of fetal heart rate patterns during labor. V. Variable decelerations. American Journal of Obstetrics and Gynecology, 132, 791-805.

[11] Krebs, H.B., Petres, R.E. and Dunn, L.J. (1983) Intrapartum fetal heart rate monitoring. VIII. Atypical variable decelerations. American Journal of Obstetrics and Gynecology, 145, 297-305. 
[12] National Institute of Child Health and Human Development (NICHD) Research Planning Workshop (1997) Electronic fetal heart rate monitoring: Research guidelines for interpretation. American Journal of Obstetrics and Gynecology, 177, 1385-1390.

[13] Cunningham, F.G., Leveno, K.J., Bloom, S.L., Hauth, J.C., Rouse, D.J. and Spong, C.Y. (2010) Intrapartum assessment. In: Cunningham, F.G., Leveno, K.J., Bloom, S.L., Hauth, J.C., Rouse, D.J. and Spong, C.Y., Eds., Williams Obstetrics, 23rd Edition, McGraw Hill Medical, New York, 410-433.

[14] Sholapurkar, S.L. (2012) The conundrum of vanishing early decelerations in British obstetrics, a step backwards? Detailed appraisal of British and American classifications of fetal heart rate decelerations-Fallacies of emphasis on waveform and putative aetiology. Journal of Obstetrics \& Gynaecology, 32, 505-511. doi:10.3109/01443615.2012.689029

[15] Miller, D.A. and Miller, L.A. (2012) Electronic fetal heart rate monitoring: Applying principles of patient safety. American Journal of Obstetrics and Gynecology, 206, 278-283. doi:10.1016/j.ajog.2011.08.016

[16] Freeman, R.K., Garite, T.H. and Nageotte, M.P. (2003) Fetal heart rate monitoring. 3rd Edition, Lippincott Williams and Wilkins, Philadelphia.

[17] OFSEP. Online Fetal Surveillance Education Programme. The Royal Australian and New Zealand College of Obstetricians and Gynaecologists. Accessed 12th February 2013.

[18] Hibbard, B.M. (1988) Assessment of fetal condition during labour. In: Hibbard, B.M., Ed., Principles of Obstetrics, Butterworth \& Co. (Publishers) Ltd., London, 472489.

[19] Malvern, J. (1989) The clinical management of labour. In: Turnbull, A. and Chamberlain, G., Eds., Obstetrics, Churchill Livingstone, London, 713-723.

[20] Ritchie, J.W.K. (1986) Fetal surveillance. In: Whitfield, C.R., Ed., Dewhurst's Textbook of Obstetrics and Gynecology for Postgraduates, 4th Edition, Blackwell Scientific Publications, Oxford, 442-462.

[21] Williams, J. and Blanchard, J. (1996) Suspicious and pathological fetal heart traces. In: Electronic Monitoring of Fetal Heart, Books for midwives Press, Hale, 24-42.

[22] Edington, P.T., Sibanda, J. and Beard, R.W. (1975) Influence on clinical practice of routine intra-partum fetal monitoring. British Medical Journal, 3, 341-343. doi:10.1136/bmj.3.5979.341

[23] Steer, P.J., Little, D.J., Lewis, N.L., Kelly, M.C. and Beard, R.W. (1976) The effect of membrane rupture on fetal heart rate in induced labour. BJOG: An International Journal of Obstetrics \& Gynaecology, 83, 454-459. doi:10.1111/j.1471-0528.1976.tb00865.x

[24] Royal College of Obstetricians and Gynaecologists (2001) Clinical Effectiveness Support Unit. The use of electronic fetal monitoring. The use and interpretation of cardiotocography in intrapartum fetal surveillance. Evidence based Clinical Guideline No. 8. RCOG Press, London.

[25] Society of Obstetricians and Gynaecologists of Canada
(SOGC) (2007) Fetal health surveillance: Antepartum and intrapartum consensus guideline. Clinical Practice Guideline No 197. Accessed September 20, 2012.

[26] Standing, S., Ellis, H., Healy, J.C., Johnson, D. and Williams, A. (2005) Chapter-Implantation, placentation, pregnancy and parturition. In: Gray's Anatomy-The Anatomical Basis of Clinical Practice, 39th Edition, Elsevier, Churchill Livingstone Ltd., London, 1139-1352.

[27] Cunningham, F.G., Gant, N.F., Leveno, K.J., Gilstrap III, L.C., Hauth, J.C. and Wenstrom, K.D. (2001) ChapterThe placenta and fetal membranes. In: Cunningham, F.G., Gant, N.F., Leveno, K.J., Gilstrap III, L.C., Hauth, J.C., and Wenstrom, K.D., Eds., William's Obstetrics, 21st Edition, McGraw Hill Medical, New York, 85-108.

[28] Lee, C.Y., Di Loreto, P.C. and O’Lane, J.M. (1975) A study of fetal heart rate acceleration patterns. Obstetrics and Gynecology, 45, 142-146.

[29] eFM. Fetal Heart Rate Monitoring (2012) An e-learning resource improving the interpretation of EFM in labour and its subsequent management. Royal College of Obstetricians and Gynaecologists and the Royal College of Midwives, London. Accessed February 15, 2012.

[30] Gibb, D. and Arulkumaran, S. (2007) Control of fetal heart rate and NICE guidelines. In: Gibb, D. and Arulkumaran, S., Eds., Fetal Monitoring in Practice, 3rd Edition, Churchill Livingstone, London, 27-44.

[31] Giussani, D.A., Unno, N., Jenkins, S.L., Wentworth, R.A., Derks, J.B., Collins, J.H. and Nathanielsz, P.W. (1997) Dynamics of cardiovascular responses to repeated partial umbilical cord compression in late-gestation sheep fetus. The American Journal of Physiology, 273, H2351H2360.

[32] Itskovitz, J., LaGamma, E.F. and Rudolph, A.M. (1983) Heart rate and blood pressure responses to umbilical cord compression in fetal lambs with special reference to the mechanism of variable deceleration. American Journal of Obstetrics and Gynecology, 147, 451-457.

[33] Yasui, T., Kimura, Y., Murotsuki, J., Watanabe, T., Koshino, T. and Okamura, K. (2002) V-shaped deceleration differs in the pattern of carotid blood flow from variable deceleration provoked by cord compression. Journal of Perinatal Medicine, 30, 257-264. doi:10.1515/JPM.2002.036

[34] Bennet, L., Westgate, J.A., Liu, Y.C., Wassink, G. and Gunn, A.J. (2005) Fetal acidosis and hypotension during repeated umbilical cord occlusions are associated with enhanced chemoreflex responses in near-term fetal sheep. Journal of Applied Physiology, 99, 1477-1482. doi:10.1152/japplphysiol.00431.2005

[35] Mallard, E.C., Gunn, A.J., Williams, C.E., Johnston, B.M. and Gluckman, P.D. (1992) Transient umbilical cord occlusion causes hippocampal damage in the fetal sheep. American Journal of Obstetrics and Gynecology, 167, 1423-1430.

[36] Cahill, A.G., Roehl, K.A., Odibo, A.O. and Macones, G.A. (2012) Association of atypical decelerations with acidemia. Obstetrics and Gynecology, 120, 1387-1393.

[37] Cahill, A.G., Roehl, K.A., Odibo, A.O. and Macones, G.A. (2012) Association and prediction of neonatal aci- 
demia. American Journal of Obstetrics and Gynecology, 207, 206.e1-206.e8.

[38] K2 Fetal Monitoring Training System (2009) K2 Medical Systems, Plymouth, United Kingdom.

\section{http://www.k2ms-university.com}

[39] Hankins, G.D., Snyder, R.R., Hauth, J.C., Gilstrap 3rd, L.C. and Hammond, T. (1987) Nuchal cords and neonatal outcome. Obstetrics and Gynecology, 70, 687-691. 